\title{
High density cluster jet target for storage ring experiments
}

\author{
A. Täschner ${ }^{\mathrm{a}, *}$, E. Köhler ${ }^{\mathrm{a}}$, H.-W. Ortjohann ${ }^{\mathrm{a}}$, A. Khoukaz ${ }^{\mathrm{a}}$ \\ ${ }^{a}$ Institut für Kernphysik, Westfälische Wilhelms-Universität Münster, D-48149 Münster, Germany
}

\begin{abstract}
The design and performance of a newly developed cluster jet target installation for hadron physics experiments are presented which, for the first time, is able to generate a hydrogen cluster jet beam with a target thickness of above $10^{15}$ atoms $/ \mathrm{cm}^{2}$ at a distance of two metres behind the cluster jet nozzle. The properties of the cluster beam and of individual clusters themselves are studied at this installation. Special emphasis is placed on measurements of the target beam density as a function of the relevant parameters as well as on the cluster beam profiles. By means of a time-of-flight setup, measurements of the velocity of single clusters and velocity distributions were possible. The complete installation, which meets the requirements of future internal fixed target experiments at storage rings, and the results of the systematic studies on hydrogen cluster jets are presented and discussed.
\end{abstract}

Keywords:

internal target, cluster jet target, hydrogen clusters, Laval nozzle

\section{Introduction}

Internal target beam facilities at storage rings play an important role in atomic, nuclear, and particle physics. They allow for precision experiments in combination with high luminosities and low physical background. In contrast to standard fixedtarget installations, where the beam hits a target only once, in storage ring experiments the accelerated beam particles traverse the target material after each round-trip, typically with a repetition rate of $10^{5}-10^{6} \mathrm{~s}^{-1}$. In order to make the best possible use of the stored beam the stationary target must be rather thin, i.e. typically only $10^{12}-10^{15}$ nuclei $/ \mathrm{cm}^{2}$ thick. This is particularly important if beams of rare particles, such as antiprotons, are used. In these cases the limited production rate should not be exceeded by the consumption rate. Further limitations of the interaction rate are introduced by the detector setup and the data acquisition system. Such a thin target can be realized by gas at rest, or beams of gas, clusters, or pellets. Depending on the experimental situation, at each passage through the interaction point only a fraction of the stored particles interacts with the target while the other projectiles remain in the beam. However, some beam losses will happen through scattering. Single scattering losses, where beam particles are kicked out of the acceptance in one collision, can be minimized by a large angular acceptance at the interaction point. By using beam cooling devices, such as electron or stochastic cooling, multiple scattering losses accumulated over many turns can be compensated. Depending on the conditions, the injected accelerator beam can be used for cycle times from minutes to hours.

\footnotetext{
${ }^{*}$ Corresponding author.

Email address: taschna@uni-muenster.de (A. Täschner)
}

The luminosity $L$ of an internal target experiment is given by the number of circulating beam particles $N_{\mathrm{C}}$, the revolution frequency $f$ of the stored particles, and the target thickness $n_{\mathrm{t}}$ :

$$
L=n_{\mathrm{t}} N_{\mathrm{C}} f
$$

Typical values for these parameters in hadron physics experiments are about $10^{10}-10^{11}$ circulating particles and an areal target thickness on the order of $10^{12}-10^{15}$ atoms $/ \mathrm{cm}^{2}[5]$.

Although the requirements for the internal targets might differ from experiment to experiment, some of them are more general: The target thickness should be constant over time to avoid luminosity fluctuations in the data acquisition system. Furthermore the target material should show a homogeneous density distribution at the interaction point. It should be of highest purity in order to avoid background reactions originating from unwanted target isotopes. The areal target thickness should be adjustable over a wide range in order to be able to match the requirements of the physics programme and the specific detection system. In the absence of vertex tracking detectors, the track and momentum reconstruction for ejectiles is aided by a precise knowledge of the interaction region. This commonly requires the geometrical target extension to be as small as possible with a sharp boundary between the target and the surrounding vacuum. The above mentioned requirements might, of course, be very challenging if a special element has to be used as a target material.

In case of experiments where only a low target thickness, on the order of $10^{12}$ atoms $/ \mathrm{cm}^{2}$, is desired or needed (supersonic) gas jets, produced by the expansion of gases through fine nozzles into vacuum, are typically used. While, in principle, all gaseous target materials could be used and the target thickness would display no time structure, this type of target has the disadvantage of generating a target beam with a large geometrical divergence. In addition, the spatial target density distribution is 


\begin{tabular}{l|ccc|c} 
& $\begin{array}{c}\text { PROMICE/WASA } \\
\text { (CELSIUS) }\end{array}$ & $\begin{array}{c}\text { E835 } \\
\text { (FERMILAB) }\end{array}$ & $\begin{array}{c}\text { ANKE and COSY-11 } \\
\text { (COSY) }\end{array}$ & $\begin{array}{c}\text { Münster } \\
\text { (this work) }\end{array}$ \\
\hline nozzle diameter & {$[1]$} & {$[3]$} & {$[4]$} & $11-28 \mu \mathrm{m}$ \\
gas temperature & $20-35 \mathrm{~K}$ & $15-40 \mathrm{~m}$ & $11-16 \mu \mathrm{m}$ & $22-35 \mathrm{~K}$ \\
gas pressure & $1.4 \mathrm{bar}$ & $<8 \mathrm{bar}$ & $18 \mathrm{bar}$ & $>18 \mathrm{Kar}$ \\
distance from nozzle & $0.325 \mathrm{~m}$ & $0.26 \mathrm{~m}$ & $0.65 \mathrm{~m}$ & $2.1 \mathrm{~m}$ \\
target thickness & $1.3 \times 10^{14} \mathrm{~cm}^{-2}$ & $>2 \times 10^{14} \mathrm{~cm}^{-2}$ & $\gg 10^{14} \mathrm{~cm}^{-2}$ & $\geq 10^{15} \mathrm{~cm}^{-2}$
\end{tabular}

Table 1: Comparison of the typical operation parameters and achieved target densities of different cluster jet targets. The densities observed with the Münster setup (right hand column) are obtained at a distance of more than two metres behind the nozzle.

locally homogeneous, apart from the distinct structures caused by shock fronts in the immediate vicinity of the nozzle. One major disadvantage of this type of target is that the nozzle has to be placed close to the interaction point ( $\ll 1 \mathrm{~m}$ ) if high target densities $\left(\geq 10^{12}\right.$ atoms $/ \mathrm{cm}^{2}$ ) are required. This commonly introduces a high gas load to the storage ring vacuum.

On the other hand, if a high effective target thickness of up to $10^{16}$ atoms $/ \mathrm{cm}^{2}$ is desired, a pellet target [2] can be used. In this kind of target micrometer-size frozen spheres, with an individual target thickness on the order of e.g. $10^{20}$ atoms $/ \mathrm{cm}^{2}$, are generated from the target materials. The use of such microspheres introduces a very distinct time structure in the target beam. The mean effective target thickness can be modified, either by varying the distance of the pellets, or by changing the pellet size. This leads to changes in the target thickness of up to an order of magnitude. Details about this type of target can be found in Ref. [6].

In contrast, cluster jet targets provide a freely adjustable target thickness of up to about $10^{15}$ atoms $/ \mathrm{cm}^{2}$ [7] even at larger distances from the nozzle. They produce small nanoparticles from cooled gases or liquids via expansion in Laval-type nozzles, by either condensation of the gas or by breaking up the liquid into a spray of tiny droplets. These clusters typically consist of $10^{3}-10^{6}$ molecules [8, 9]. Different to pellet targets, the random nature of the cluster production introduces a homogeneous spatial distribution and no distinct time structure. All gaseous target materials can be used for this kind of target. The cross-section of the cluster beam at the interaction point exhibits a well defined boundary so that the interaction region is well defined. This property has the important consequence that the beam can pass several metres through an ultra high vacuum with constant angular divergence defined by the orifices used for the cluster beam preparation.

Future internal fixed-target experiments at storage rings require predominantly high density hydrogen or deuterium targets as effective proton and deuteron/neutron beams. Due to their various advantages, cluster jet targets are well established in high precision experiments [3, 4, 10]. Typical operation parameters of some of the targets used at the CELSIUS ring [1], at the Fermilab Antiproton Accumulator [3], and at COSY [4, 11] are listed in Table 1. Due to the finite angular divergence of the cluster beam, the volume target density decreases quadratically with the distance from the nozzle. At these experiments the distances between the cluster production nozzles and the interaction points range between $0.26 \mathrm{~m}$ and $0.65 \mathrm{~m}$, resulting in hydrogen target densities of several $10^{14}$ atoms $/ \mathrm{cm}^{2}$. Future facilities for hadron physics experiments will be typically $4 \pi$ detectors, where the interaction point between the stored beam and the target beam is surrounded by onion-like layers of detectors embedded in a magnetic field, allowing a momentum reconstruction of the charged ejectiles. One prominent example of such an upcoming installation is the planned PANDA detector at FAIR in Darmstadt [12]. The proposed detector concept requires that the target source must be mounted outside the coils of the magnet and its surrounding iron yoke, with the result that the distance from the nozzle to the interaction point will increase from around $0.5 \mathrm{~m}$, as in current targets, to more than $2 \mathrm{~m}$. For cluster jet targets, this increase leads, for geometrical reasons, to a decrease in the target thickness by approximately one order of magnitude. Because of this effect, new studies on higher cluster jet target densities were started in Münster. We here present a newly developed cluster target device which, for the first time, is able to provide hydrogen cluster beam densities on the order of $10^{15}$ atoms $/ \mathrm{cm}^{2}$ at a distance of more than two metres from the nozzle. This achievement opens the way to use the broad advantages of cluster jet beams in future hadron physics experiments with large $4 \pi$ detectors.

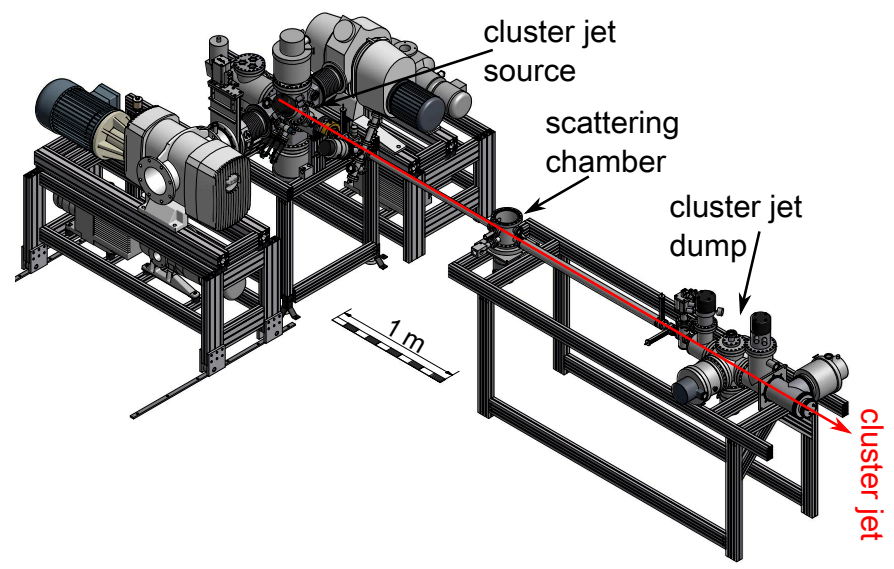

Figure 1: View of the complete setup, with the cluster source and its pumping system on the left, the scattering chamber with beam diagnostics in the middle, and the cluster beam dump on the right. 


\section{Experimental setup}

A complete target station was designed and built up which allows one to test improved cluster sources in a geometry which matches that of an internal storage ring experiment with a $4 \pi$ detector. Since the dimensions of the future PANDA experiment are specified [12], the distances relevant to this target station were used for the setup. In Fig. 1 a CAD view of the experimental setup is shown, with the cluster source and its pumping system on the left, a scattering chamber with beam diagnostics in the middle, and the cluster beam dump on the right. The distance of $2.1 \mathrm{~m}$ between the nozzle inside the cluster source and the centre of the scattering chamber is the same as the one planned for the PANDA experiment. Furthermore, the distance between the scattering chamber and the cluster beam dump also matches the one from PANDA. In this way the data obtained can be directly translated to the situation in the planned storage ring experiment.

In the source the clusters are generated and the shape of the cluster jet is prepared. The cluster source consists of different vacuum chambers forming a differential pumping system in

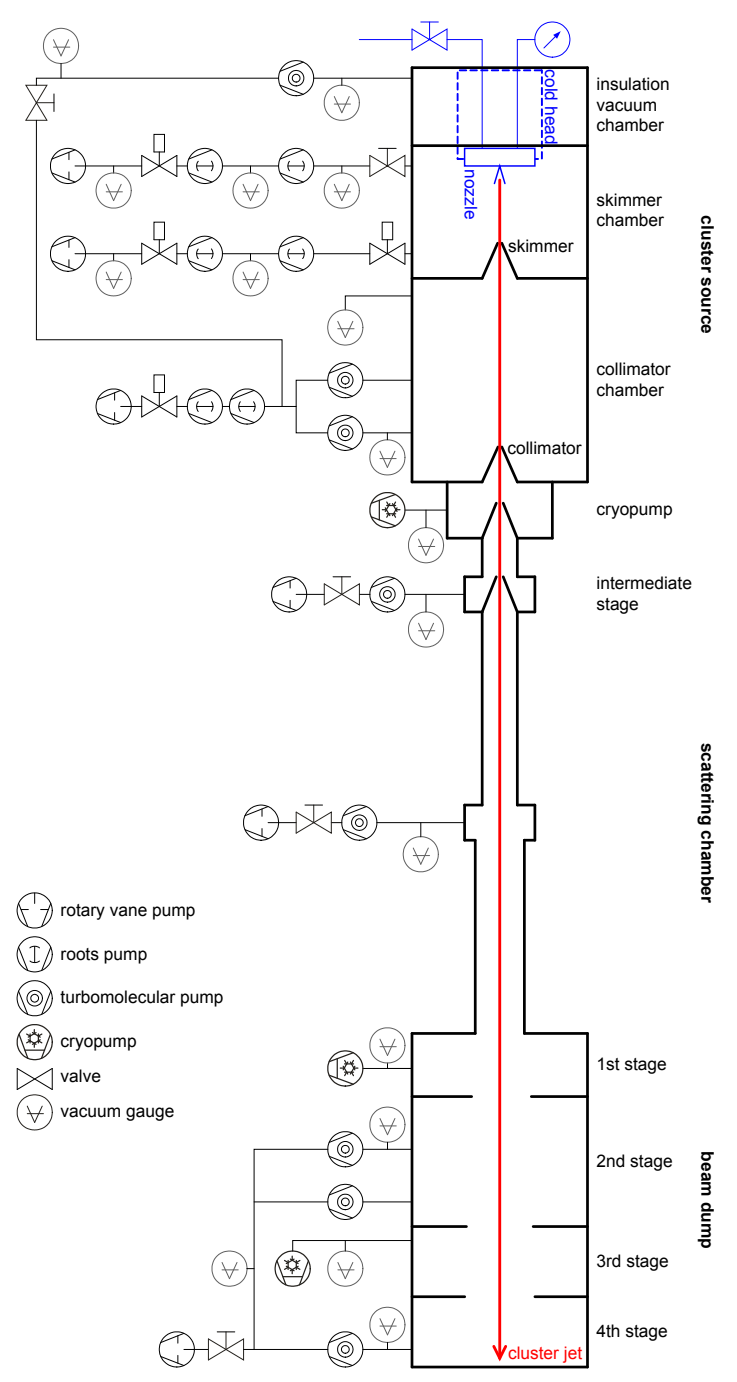

Figure 2: The vacuum system of the Münster cluster jet target setup. order to reduce the gas flow from the source into the interaction region. This is of great importance in an internal storage ring experiment in order to guarantee a low residual gas load on the accelerator beam line, as well as to minimize reactions with the background gas. The scattering chamber installed is equipped with a beam diagnostic system for the determination of target thickness and geometry. The attached cluster beam dump is designed to catch the cluster jet beam and to minimize the back flow of gas to the scattering chamber. In Fig. 2 the complete design of the vacuum system of the target setup is shown. The individual parts will be discussed in the following sections. A list of the most important pumps, and typical pressures observed during the experiments in the different pumping stages, is compiled in Table 2 For the investigations presented in this work, the main emphasis was placed on achieving highest target thicknesses and the measurement of the cluster beam properties. Thus, with exception of the skimmer and the collimator, the orifices connecting neighbouring vacuum chambers have been chosen rather generously in diameter in order to avoid hitting them when changing the cluster beam diameter through the use of different collimator sizes. Therefore, the vacuum conditions presented here are expected to be improved significantly when the cluster beam size is fixed and appropriate orifice diameters are chosen.

\begin{tabular}{|c|c|c|}
\hline Pumping stage & Pumps & Typical pressure \\
\hline $\begin{array}{l}\text { Insulation } \\
\text { vacuum chamber }\end{array}$ & $\begin{array}{l}\text { Turbo pump: 3701/s } \\
\text { (Leybold Turbovac 361) }\end{array}$ & $10^{-5} \mathrm{mbar}$ \\
\hline $\begin{array}{l}\text { Skimmer } \\
\text { chamber }\end{array}$ & $\begin{array}{l}\text { Roots pump: } 3000 \mathrm{~m}^{3} / \mathrm{h} \\
\text { (Leybold Ruvac RA3001) } \\
\text { Roots pump: } 2000 \mathrm{~m}^{3} / \mathrm{h} \\
\text { (Leybold Ruvac WSL2001) }\end{array}$ & $8 \times 10^{-2} \mathrm{mbar}$ \\
\hline $\begin{array}{l}\text { Collimator } \\
\text { chamber }\end{array}$ & $\begin{array}{l}\text { Turbo pump: } 2 \times 9001 / \mathrm{s} \\
\text { (Leybold Turbovac } 1000 \mathrm{C} \text { ) }\end{array}$ & $2 \times 10^{-4} \mathrm{mbar}$ \\
\hline Cryopump stage & Münster type cryopump & $2 \times 10^{-5} \mathrm{mbar}$ \\
\hline $\begin{array}{l}\text { Intermediate } \\
\text { stage }\end{array}$ & $\begin{array}{l}\text { Turbo pump: } 1101 / \mathrm{s} \\
\text { (Leybold Turbovac 150) }\end{array}$ & $10^{-5}$ mbar \\
\hline $\begin{array}{l}\text { Scattering } \\
\text { chamber }\end{array}$ & $\begin{array}{l}\text { Turbo pump: } 3401 / \mathrm{s} \\
\text { (Leybold Turbovac 360) }\end{array}$ & $3 \times 10^{-5} \mathrm{mbar}$ \\
\hline 1st beam dump & Münster type cryopump & $6 \times 10^{-6} \mathrm{mbar}$ \\
\hline 2nd beam dump & $\begin{array}{l}\text { Turbo pump: } 9001 / \mathrm{s} \\
\text { (Leybold Turbovac } 1000 \mathrm{C} \text { ) } \\
\text { Turbo pump: } 3701 / \mathrm{s} \\
\text { (Leybold Turbovac } 361 \mathrm{C} \text { ) }\end{array}$ & $7 \times 10^{-6} \mathrm{mbar}$ \\
\hline 3rd beam dump & Münster type cryopump & $3 \times 10^{-5} \mathrm{mbar}$ \\
\hline 4th beam dump & $\begin{array}{l}\text { Turbo pump: } 9001 / \mathrm{s} \\
\text { (Leybold Turbovac } 1000 \mathrm{C} \text { ) }\end{array}$ & $8 \times 10^{-5} \mathrm{mbar}$ \\
\hline
\end{tabular}

Table 2: Vacuum pumping system of the cluster jet target with pressures for operation at highest target thickness and therefore highest gas flow through the nozzle. 


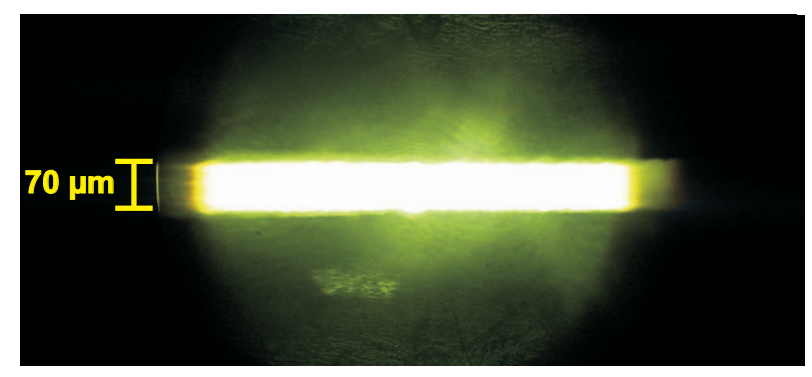

Figure 3: Microscopic view of the orifice of a slit collimator produced by a laser-cut method.

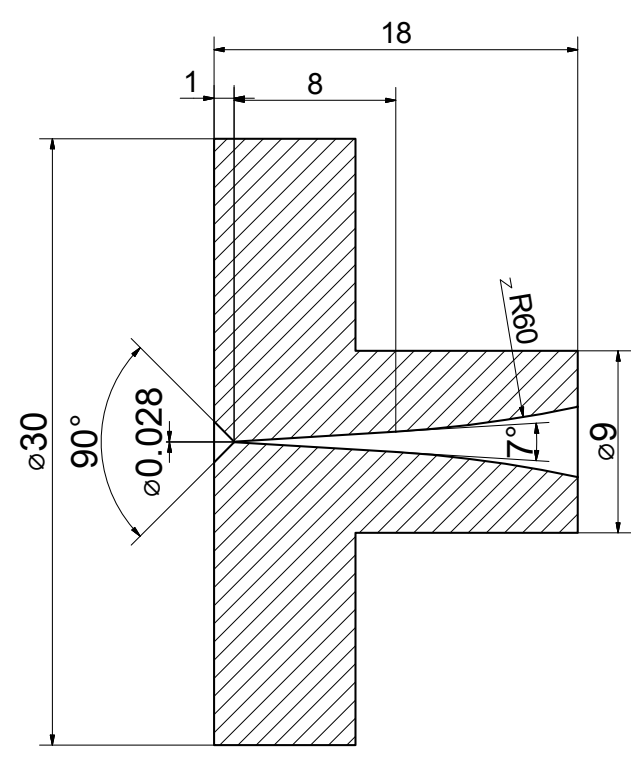

Figure 4: Cross-section of the copper Laval nozzle used. This was manufactured in the CERN workshop.

More detailed investigations on the minimization of the residual gas flow to the scattering chamber are currently under evaluation [13]. Apart from the standard way to suppress the gas background by reducing the orifice diameters between the vacuum chambers, there is also the possibility to use cluster beams with optimized cross-sections. At a given target thickness the flow rate of the cluster beam can be minimized by reducing the beam size transverse the direction of the ion or electron beam. Assuming an ion beam size of one millimetre and a cluster beam size of ten millimetres the gas flow can be reduced by almost one magnitude using a cluster beam with a rectangular cross-section of $1 \mathrm{~mm} \times 10 \mathrm{~mm}$ instead of a circular one. This concept has been proposed before, e.g. for the UA6 experiment at CERN using a polarized atomic beam target [22], and used successfully with a cluster jet target at the COSY11 experiment [23]. First tests on this idea have been carried out at the cluster target installation by using specially shaped collimators produced by laser cutting. In Fig. 3 a microscopic view of such an orifice is shown with a size of $70 \mu \mathrm{m} \times 860 \mu \mathrm{m}$, resulting in a cluster beam with a length of $\sim 13 \mathrm{~mm}$ in the ion beam direction and $\sim 1.5 \mathrm{~mm}$ perpendicular to it.

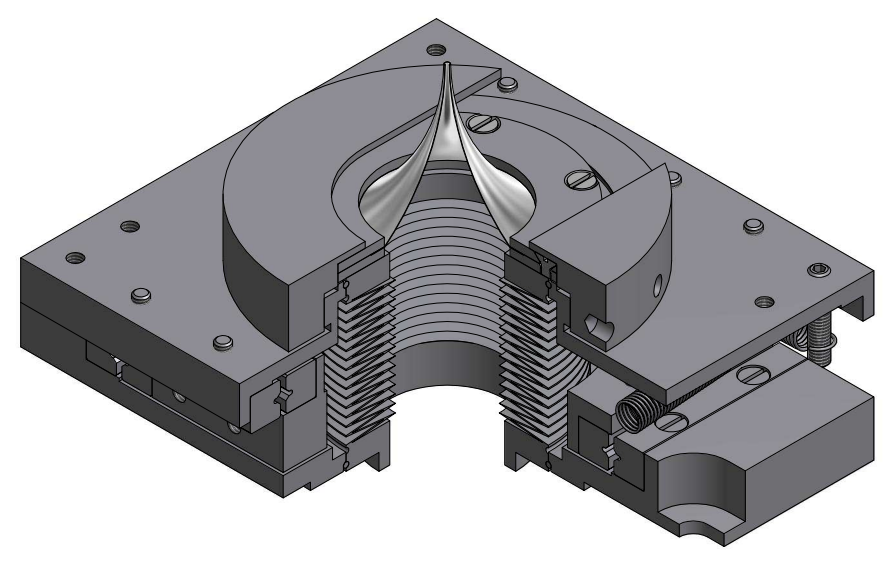

Figure 5: Cross-section of the $X-Y$ table used to move the skimmers. The edgewelded bellow separates the different vacuum stages in front and behind the skimmer.

\subsection{Cluster source}

The setup is optimized for PANDA at HESR where mostly hydrogen will be used as target material. The clusters are produced by the expansion of cooled hydrogen gas or liquid through a Laval nozzle into a vacuum chamber. The one used, which is illustrated in Fig. 4. was manufactured in the CERN workshop from copper and has a minimum diameter of approximately $28 \mu \mathrm{m}$. The hydrogen gas, with pressures of up to 20 bar, has to be purified in order to prevent clogging of the cold nozzle by frozen impurities. For this purpose a commercial purification system (Johnson-Matthey HE20) based on a palladium membrane is used. This achieves a purity level of 9.0, which allows for continuous operation over weeks without any blocking of the nozzle. In addition a micro filter with a pore size of $0.5 \mu \mathrm{m}$ in the gas input line stops solid particles from entering the gas system. The gas is cooled by a dual stage GiffordMcMahon-type cold head (Leybold COOLPOWER $10 \mathrm{MD}$ ). The first stage has a cooling power of $110 \mathrm{~W}$ at $80 \mathrm{~K}$ and the second stage $18 \mathrm{~W}$ at $20 \mathrm{~K}$. With this device, and a $50 \mathrm{~W}$ heating cartridge, the temperature of the fluid directly before the nozzle can be adjusted by using a temperature controller (LakeShore Model 331S) in the operational region between 19 and $50 \mathrm{~K}$. In order to minimize the heat transfer by heat conductance from the walls of the vacuum chamber (room temperature) to the two stages of the cold head, the new design of the cluster source embeds the cold head in a separate vacuum chamber (insulation vacuum chamber) which has no direct connection to the vacuum in the other chambers of the cluster source.

The cold hydrogen passes the Laval nozzle, where the clusters are produced during the expansion of the fluid into an adjacent vacuum chamber, the skimmer chamber. Depending on the operational parameters, only a small fraction of the gas produces clusters. From this cluster jet a certain part, which depends on the experimental requirements, is selected by skimmers. Thus most of the gas flow through the nozzle, i.e. approximately up to $80 \mathrm{mbar} \cdot 1 / \mathrm{s}$ hydrogen gas, has to be pumped away from this chamber. Since it was observed that vacuum pressures above $\approx 10^{-1}$ mbar lead to a loss of target 


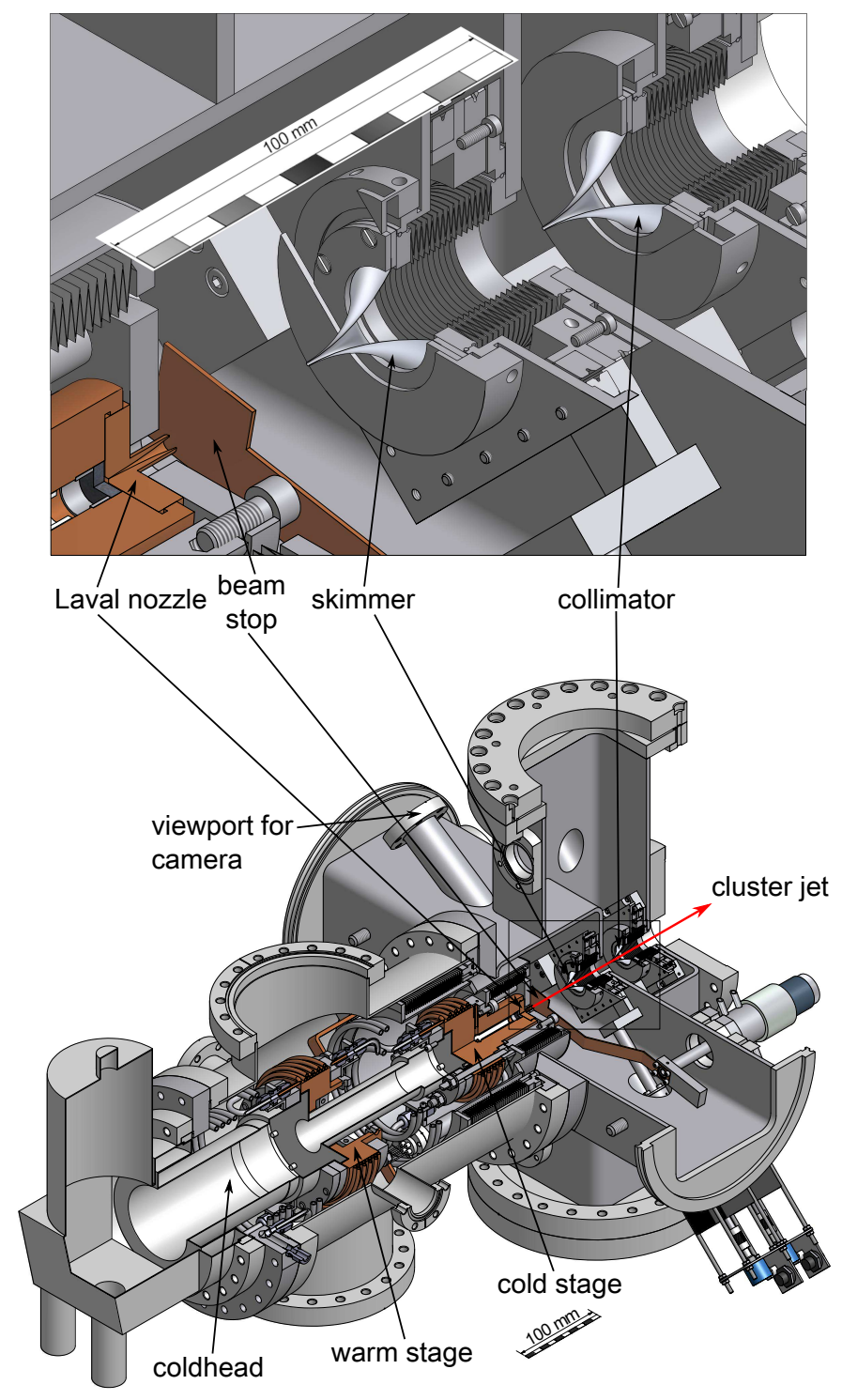

Figure 6: View of the complete new cluster source and an expanded view of the main parts of the source.

thickness [14], the pumping speed of the pumps used is chosen in order to match this requirement. Therefore, the two pumping systems shown in Fig. 2, each consisting of two roots and one rotary vane pump with a total pumping speed of $5000 \mathrm{~m}^{3} / \mathrm{h}$, are used to evacuate this vacuum chamber.

The clusters produced can be separated from the surrounding gas by using a trumpet-shaped skimmer with an opening diameter of the orifice of $0.5 \mathrm{~mm}$ (Beam Dynamics Incorporation). A second skimmer, the collimator, is mounted in an adjacent vacuum chamber. The collimator further reduces the residual gas flow into the accelerator and defines the shape and size of the cluster jet beam at the interaction point. For the data presented here, a collimator with a round orifice with an opening of $0.7 \mathrm{~mm}$ was used. Due to the limited gas conductance of the skimmer, the vacuum chamber where the collimator is located can be pumped by two turbomolecular pumps. Both the skimmer and the collimator are mounted on step-motor-driven

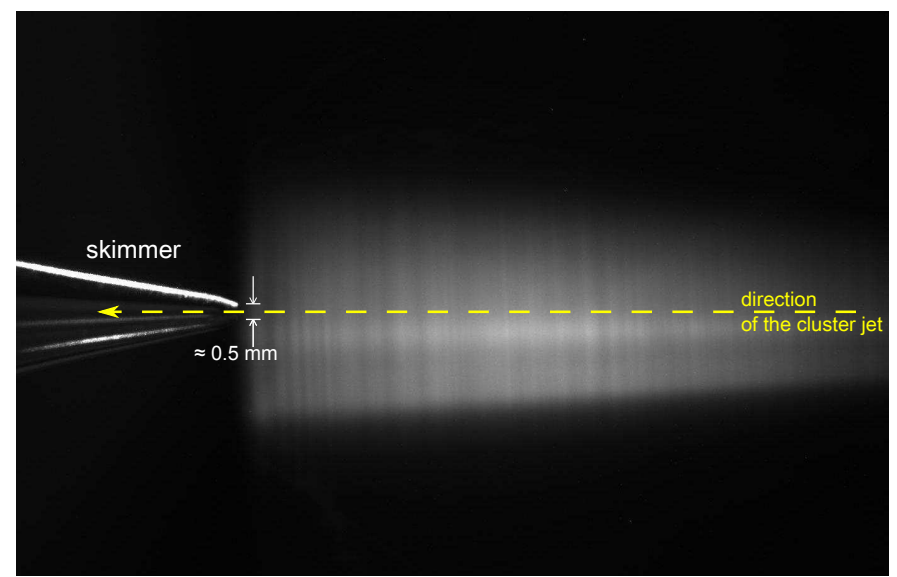

Figure 7: Photograph of the cluster jet taken in the skimmer chamber. The clusters are produced in the nozzle which is situated to the right, outside of the range of the camera. Visible is the skimmer tip on the left together with the cluster jet, illuminated by a laser beam. At this stage the opening angle of the cluster jet beam is given by the nozzle geometry.

$X-Y$ tables displayed in Fig. 5. In this way it is possible to adjust them, e.g. for alignment purposes during operation, in both directions perpendicular to the cluster beam in steps of one micrometre with a maximum displacement of two millimetres with respect to the central axis of the setup. The complete setup is shown in Fig. 6

A motor-driven shutter (Fig. 6, "beam stop") is installed between the nozzle exit and the orifice of the first skimmer. This device allows one to stop the cluster jet beam completely in the skimmer stage on a sub-second time scale. Such a feature is of great importance for storage ring experiments where, for each cycle, the injection, acceleration and cooling of the ion beam are best done in the absence of a target stream.

The region between the nozzle and the skimmer can be observed by a camera mounted outside the vacuum at an UHV vacuum window of the skimmer chamber (Fig. 6). If the temperature and pressure of the incoming gas in front of the nozzle is chosen appropriately in a region where the hydrogen is liquid, the density can be as high that the jet is visible. An example of an image taken by this camera is shown in Fig. 7 The cluster jet, which is clearly visible in this photograph, is illuminated by an optically broadened laser beam $(P \leq 1 \mathrm{~mW})$. The vertical structures in the cluster beam are an artifact caused by the optical system. However, the clear horizontal structures in brightness of the jet correspond to density variations. It is important to note that the bright core inside the beam, which is a region of higher density, is not distributed symmetrically around the central axis of the nozzle but is directed towards the lower part of the picture. It was observed that the deflection angle of this core depends strongly on the temperature and the pressure of the fluid in front of the nozzle. At high temperatures and low pressures (e.g. $p=7$ bar and $T=35 \mathrm{~K}$ ) the structures are almost uniform and symmetrically arranged whereas at highest jet densities, corresponding to low temperatures and high pressures (e.g. $p=17 \mathrm{bar}$ and $T=25 \mathrm{~K}$ ), both the deflection and the density structure are clearly visible. This 


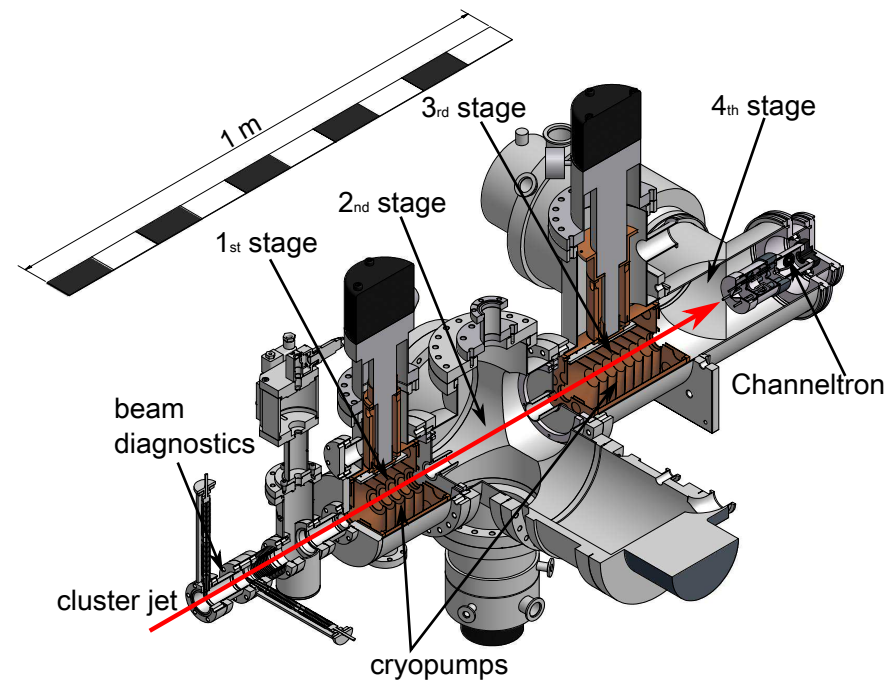

Figure 8: Beam dump of the target station based on the COSY-11 design [4]

effect is currently under investigation and indicates potential for further improvements of the cluster beam density in the scattering chamber [15, 16].

Between the collimator chamber and the beam pipe leading to the interaction point, two additional pumping stages, shown in Fig. 2, are installed in order to minimize further the gas flow from the source into the scattering chamber. The first chamber is equipped with a Münster-type cryopump [4] and the next one with a single turbomolecular pump. At the exit of both chambers, a skimmer is inserted whose inner diameter is a few millimetres larger than the cluster beam at this position to avoid interference with the cluster beam.

\subsection{Beam line and cluster beam dump}

The produced clusters are transferred through a beam pipe with an inner diameter of $38 \mathrm{~mm}$ to the interaction region where the scattering chamber is placed. In the scattering chamber, the target thickness can be measured at a distance of $2.1 \mathrm{~m}$ from the nozzle using a beam diagnostic system discussed in the next section. From this scattering chamber the clusters are transferred to the cluster beam dump. The connecting beam pipe, with an inner diameter of $66 \mathrm{~mm}$, is wider than that between the cluster source and the scattering chamber. The main design of the beam dump shown in Fig. 8 matches that from the former COSY-11 experiment [4] located at the COSY accelerator of the Forschungszentrum Jülich. This beam dump consists of four differentially pumped vacuum stages equipped with pumps of different types. The first and third stages contain cryopumps of the Münster type [4], whereas the other two stages are equipped with turbomolecular pumps. The complete system is designed to minimize the back flow of the gas from the stopped clusters into the scattering chamber. This minimization of gas load to the scattering chamber is of special importance if the equipment is used as an internal target at a storage ring. At the final stage a detector system can be installed in order to measure the cluster velocity. This system is described in detail in the following section.

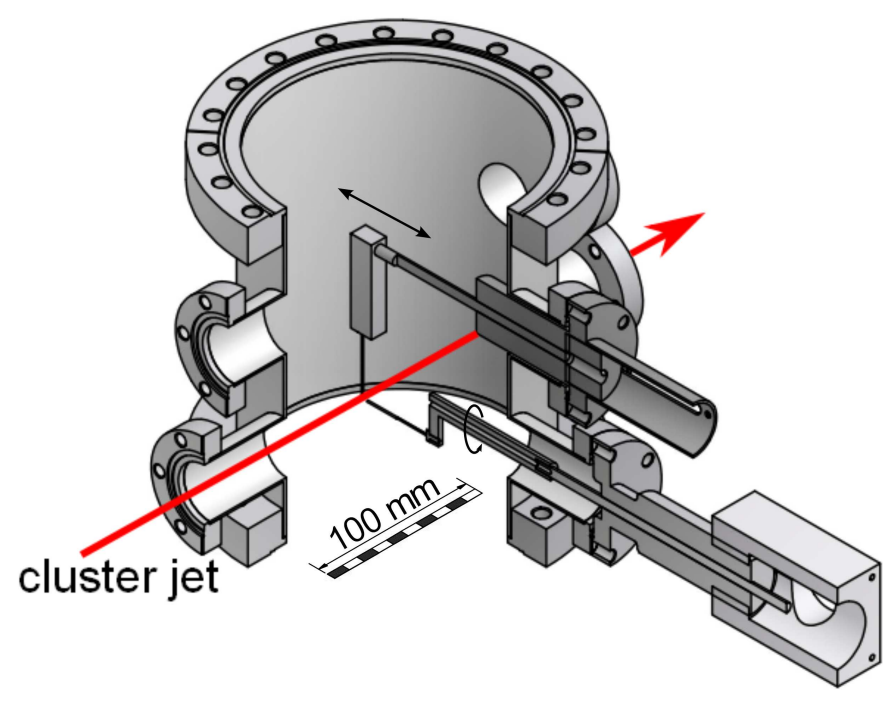

Figure 9: Scattering chamber with movable rods used for the measurement of both the absolute target thickness and the target beam profiles.

\subsection{Beam diagnostics}

One of the most important parameters which characterizes the performance of an internal target is the target thickness, which can be expressed either as volume density $\rho$ or as areal density $n_{T}$. In order to measure the target thickness at the interaction point ("PANDA geometry") a vacuum chamber shown in Fig. 9 was installed. In this scattering chamber a beam diagnostic system, consisting of two movable rods, can be used to determine the target thickness. The used rods have a diameter of one millimetre which is much smaller than the ten millimetre diameter of the cluster jet. Clusters colliding with these rods break up and generate a gas load, which increases the pressure in the vacuum chamber. In a simple model, assuming a constant density in the cross-section between rod and cluster jet, this pressure increase is directly proportional to the total hydrogen mass flow $d m / d t$ into this vacuum chamber. This allows a direct target density determination according to:

$$
\rho=\frac{d m / d t}{A v}
$$

Here $A$ corresponds to the cross-section of the cluster beam in the scattering chamber, which can be determined by beamprofile measurements using the movable rods, and $v$ is the mean velocity of the cluster jet. An example of such a beam profile measurement is shown in Fig. 10. Since this simple model cannot be used for the analysis of this measured pressure increase, the volume and areal density can be deduced by assuming a certain volume density distribution $\rho_{z}\left(x^{\prime}, y^{\prime}\right)$ at the specific distance $z$ from the nozzle. The vacuum pressure $p(x)$ as a function of the position $x$ of the rod can be calculated from:

$$
p(x)=p_{\mathrm{b}}+\frac{v R T}{S M} \int_{x-x_{0}-d / 2}^{x-x_{0}+d / 2} \mathrm{~d} x^{\prime} \int_{-\infty}^{+\infty} \mathrm{d} y^{\prime} \rho_{z}\left(x^{\prime}, y^{\prime}\right)
$$

where $v$ is the mean velocity of the clusters, $R$ the universal gas constant, $T$ the temperature of the gas, $M$ the molar mass of 


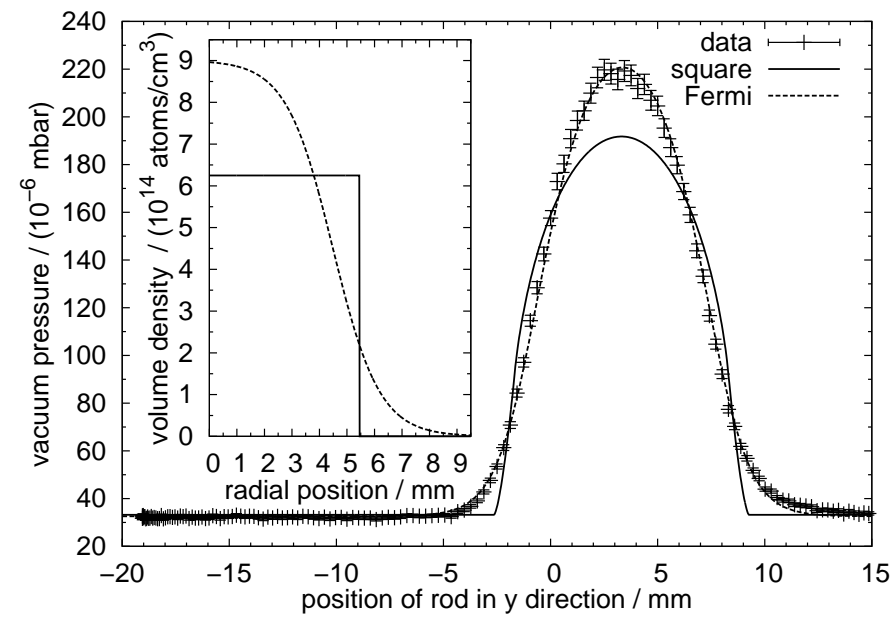

Figure 10: Measured pressure in the scattering chamber as a function of the position of the vertical rod (diameter: one millimetre) shown in Fig. 9 The solid line represents a fit assuming a homogeneous radial volume density of the scanned cluster jet with sharp boundaries while the dashed curve represents a fit to a Fermi-like density distribution expressed in Eq. 6. The insert shows the radial volume density distributions corresponding to the drawn lines in the pressure distribution.

the hydrogen molecules, $S$ the pumping speed of the vacuum pumps used to evacuate the scattering chamber, $d$ the diameter of the scanning rod (here: $d=1 \mathrm{~mm}$ ), $x_{0}$ the displacement of the maximum of the volume distribution with respect to the coordinate system of the rod, and $p_{\mathrm{b}}$ the background pressure in the chamber. By fitting Eq. (3) to the measured data, the volume density can be estimated.

In order to be able to use Eq. (3), the mean velocity of the clusters has to be known. For this purpose a time-of-flight system (TOF) shown in Fig. 11 was implemented. Behind the cluster source a pulsed electron gun [17] is mounted, whose intensity can be reduced sufficiently to guarantee single cluster ionization. In coincidence with the pulse of the electron gun, a micro-controller-based timer is started. Due to the large mass of the clusters the effect of the ionization process on the cluster velocity can be neglected and the ionized particles move with their original velocity to the stop detector located in the beam dump. Here the ionized clusters are detected by a Channeltron shown in Fig. 12 which provides the electronic signal used to

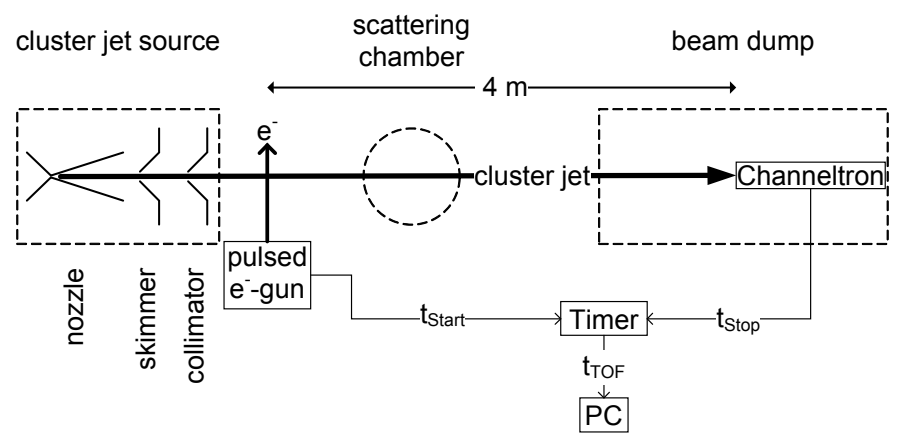

Figure 11: Schematic diagram of the time-of-flight system used to measure the velocity of individual clusters.

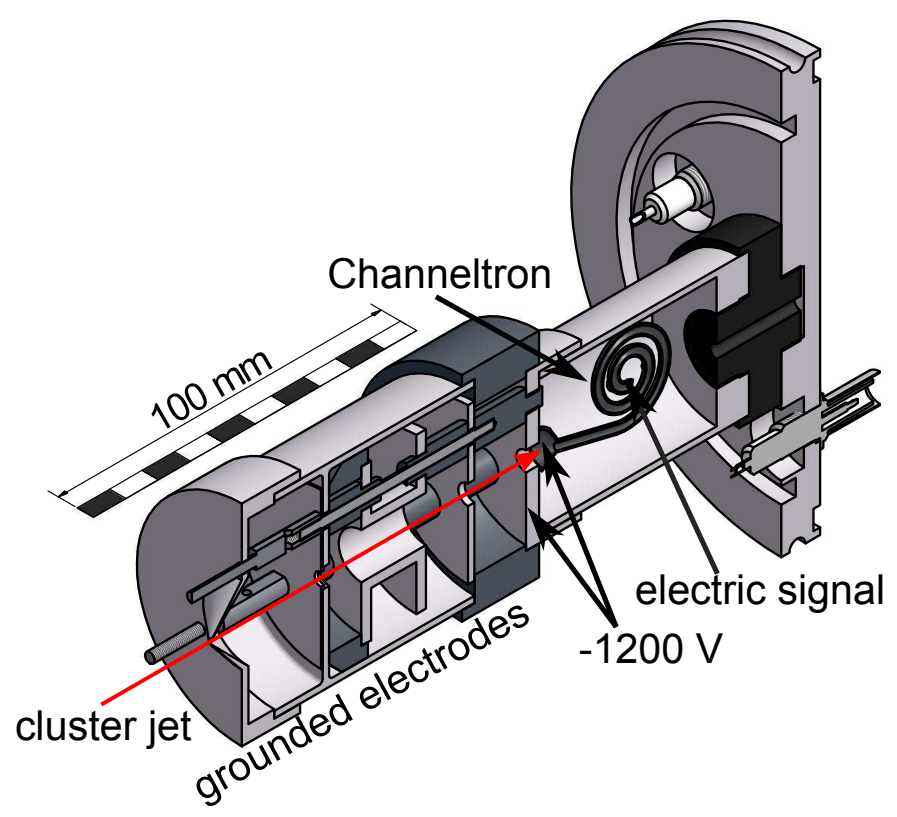

Figure 12: Detection system used for the generation of the stop signal of the TOF measurements.

stop the timer. Since neutral clusters were found to generate no signal, spectra with minimal background were obtained.

Before starting systematic velocity measurements, the system was calibrated by means of TOF measurements using ionized hydrogen gas with precisely known kinetic energy and velocity of the ions [18]. In Fig. 13 the result of such a measurement is shown. In this spectrum four peaks can be identified, corresponding to three different hydrogen ions, namely $\mathrm{H}^{+}, \mathrm{H}_{2}^{+}$, and $\mathrm{H}_{3}^{+}$, and photons emitted from the ionized gas. By using several measurements with different acceleration voltages, the time offset of the electronic system used and the length of the flight path could be determined. A length of $4.02(3) \mathrm{m}$ was obtained, which agrees well with direct distance measurements.

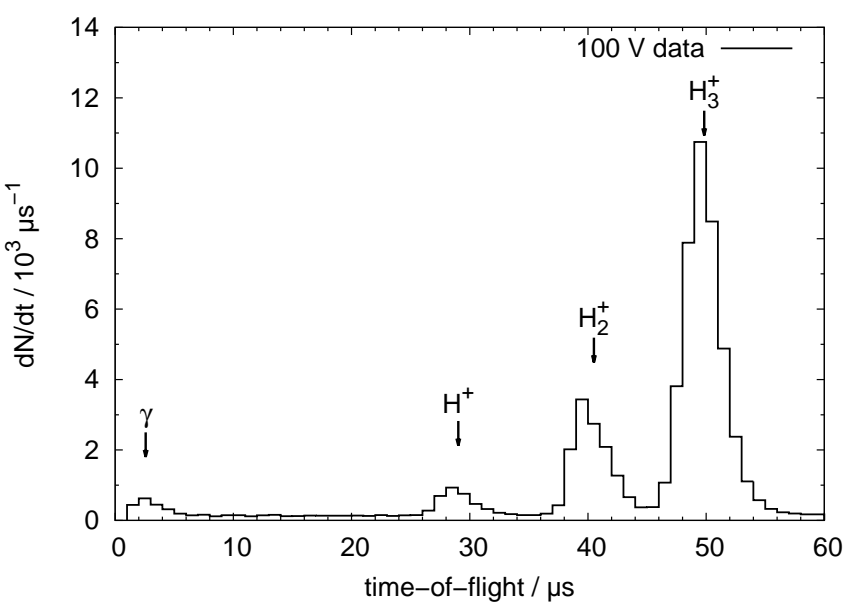

Figure 13: Time-of-flight distribution of different hydrogen ions accelerated through a voltage of $100 \mathrm{~V}$. The calibration source used to generate and accelerate the ions also emits photons that are visible in the spectrum. 


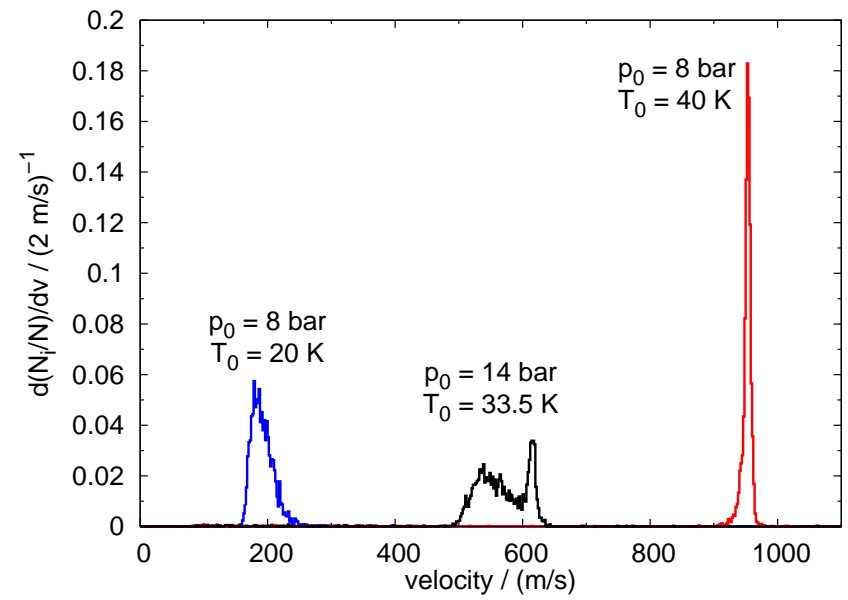

Figure 14: Three examples of velocity distributions of hydrogen clusters measured with the time-of-flight setup at different pressures $p_{0}$ and temperatures $T_{0}$ of the hydrogen before entering the nozzle.

In addition a time resolution of around three microseconds was observed. This originates from the pulse width of the electron gun, as well as from the finite width of the energy distribution of the generated ions. The time resolution is therefore better than $0.1 \%$ of the time-of-flight of a cluster, which is typically several milliseconds. By using this system, the velocity distribution could be measured and the mean velocity needed for the target thickness estimation extracted.

Examples of the measured cluster velocity spectra are shown in Fig. 14. The frequency density distribution (ordinate) shown is obtained for three examples of hydrogen temperature and pressure by dividing the number of observed clusters $N_{i}$ in the depicted velocity intervals by the total number of observed clusters $N=\sum N_{i}$ so that the integral over the displayed velocity range is equal to one. The three distributions represent different modes of operation of the cluster jet source. At the temperature and pressure of the displayed distribution with the highest mean velocity of $953 \mathrm{~m} / \mathrm{s}$, the hydrogen is gaseous in front of the nozzle. In this case the clusters are produced by condensation of the gas, leading to a narrow distribution with a width $(1 \sigma)$ of $5 \mathrm{~m} / \mathrm{s}$. The distribution with the lowest mean velocity of $191 \mathrm{~m} / \mathrm{s}$ is observed for temperatures and pressures where the hydrogen is already in a liquid phase before entering the nozzle. Obviously here the clusters are produced by break up of the liquid jet injected into vacuum. As can be seen from the spectra obtained, this production mechanism leads to a much wider velocity distribution, on the order of $16 \mathrm{~m} / \mathrm{s}$ $(1 \sigma)$. For temperatures and pressures near the phase transition (gas phase/liquid phase) preceding the nozzle, a double peak structure is observed with a narrow distribution with higher mean velocity on top of a broader distribution. This structure was already observed in Ref. [8] at higher hydrogen pressures between 20 and 120 bar and interpreted as a production of clusters from a liquid/gas mixture. A more detailed presentation of the observed distributions can be found in [18, 19]. Furthermore, quantitative numerical calculations of the mean velocities

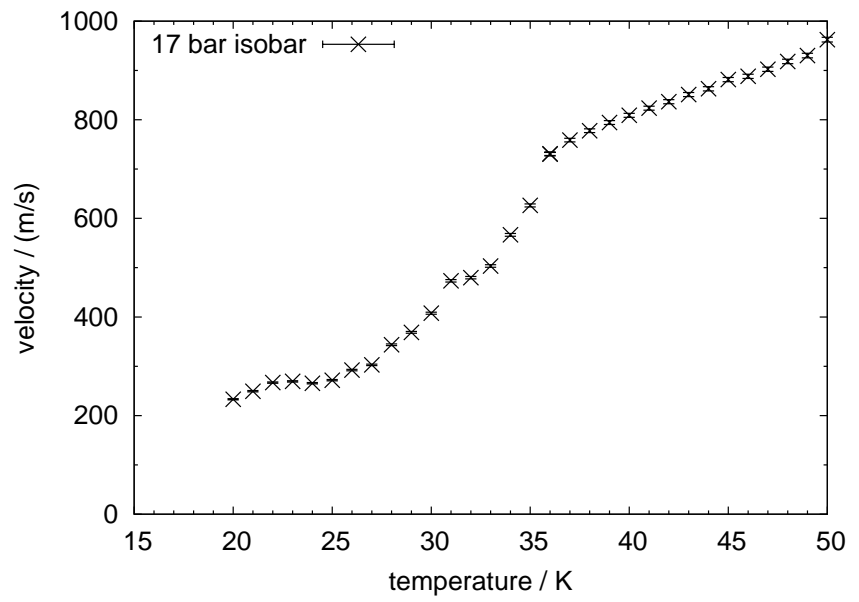

Figure 15: Mean velocity of the clusters as a function of the hydrogen temperature at the inlet of the Laval nozzle at a constant input pressure of $17 \mathrm{bar}$.

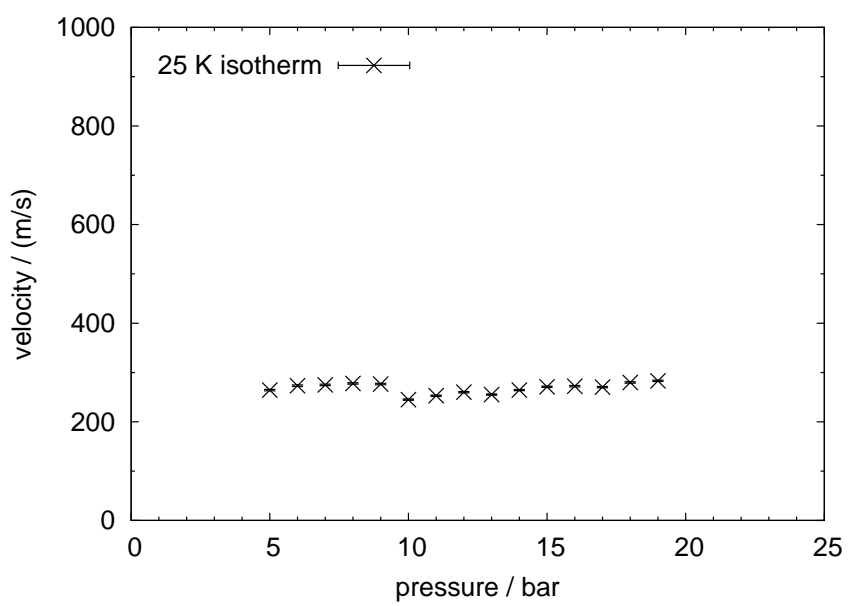

Figure 16: Dependence of the mean cluster velocity on the pressure at the inlet of the nozzle at a constant temperature of $25 \mathrm{~K}$.

as function of the pressure $p_{0}$ and temperature $T_{0}$ have been performed [18, 20].

\section{Results}

The diagnostic system described allows systematic studies on the cluster velocities and target beam thicknesses. In Fig. 15 the mean velocity is shown as a function of the temperature of the fluid before the nozzle at a constant pressure of $17 \mathrm{bar}$. Since this pressure is above the critical pressure of hydrogen, 12.964 bar [21], the phase change at the critical temperature of $33.145 \mathrm{~K}$ is continuous. The mean velocities displayed in Fig. 16 were measured at a constant temperature of $25 \mathrm{~K}$ as a function of the hydrogen pressure. In both cases the uncertainties are smaller than the symbol size. To perform systematic measurements on cluster beam densities at the same pressures and temperatures shown in these two graphs, the pressure increase was recorded as a function of the position of one of the rods in the scattering chamber. In Fig. 10 one of these pressure profiles is presented. 


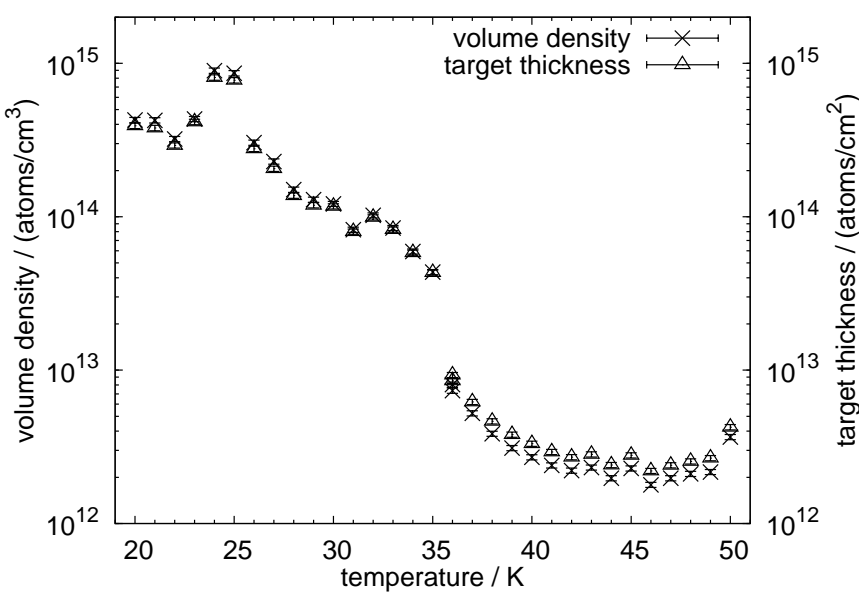

Figure 17: Volume density $\rho_{0}$ and target thickness $n_{T}$ as a function of the hydrogen temperature at the inlet of the Laval nozzle at a constant gas pressure of 17 bar, assuming a Fermi-like volume density distribution.

In order to describe the pressure profile, two different models were used which both assume a rotationally invariant volume density distribution; $\rho(r)=\rho_{0} \hat{\rho}(r)$, where $\rho_{0}$ is the maximum volume density and $r=\sqrt{x^{\prime 2}+y^{\prime 2}}$ is the distance to the centre of the distribution, and $\hat{\rho}(r=0)=1$. In this case the areal target thickness in units of number of atoms per square centimetre is given by

$$
n_{T}=\frac{2 N_{A}}{M_{a}} \int_{0}^{\infty} \rho(r) \mathrm{d} r
$$

with the molar mass of the gas atoms $M_{a}$ and the Avogadro constant $N_{A}$. The first model represents the simplest situation and assumes a homogeneous density distribution with a sharp edge at a radius $R$, where

$$
\rho_{\text {square }}(r)= \begin{cases}\rho_{0} & \text { for } r \leq R \\ 0 & \text { for } r>R .\end{cases}
$$

The solid line in Fig. 10 represents a fit to the data based on this model. The corresponding volume density distribution is shown as an inset in this figure. Obviously this simple model is not able to describe sufficiently well the observed density distribution. The second model uses an empiric ansatz with a Fermi-like function to describe the volume density distribution

$$
\rho_{\text {Fermi }}(r)=\rho_{0}\left(\exp \left(-\frac{R}{s}\right)+1\right) /\left(\exp \left(\frac{r-R}{s}\right)+1\right),
$$

with the beam radius $R$ and the parameter $s$ describing the boundary smearing of the distribution. This type of distribution leads to a prediction for the pressure increase shown by the dashed line in the figure. It is clearly seen that the data can be described with high accuracy using this kind of distribution. The reduced values of $\chi^{2} /$ ndf were found to be in the range between 0.5 and 2 .

Based on the parameters obtained by fitting the Fermi-like volume density distribution to the measured data, Figs. 17 and 18 show the values found for the maximum volume density and for the areal target thickness. At a distance of

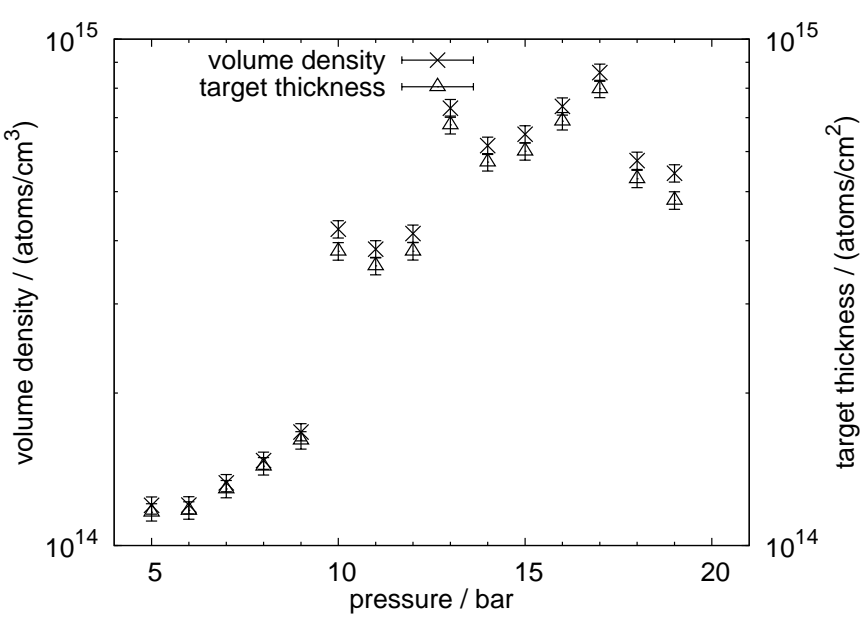

Figure 18: Dependence of the volume density $\rho_{0}$ and target thickness $n_{T}$ on the pressure at the inlet of the nozzle at a constant temperature of $25 \mathrm{~K}$, assuming a Fermi-like volume density distribution.

about two metres behind the nozzle, a target thickness of $(8.1 \pm 0.3) \times 10^{14}$ atoms $/ \mathrm{cm}^{2}$ combined with a target beam diameter $(2 R)$ of approximately nine millimetres was achieved. In agreement with earlier results, the isobaric data show a strong correlation between the target thickness and the temperature. The target thickness can be easily varied in a range between $10^{12}$ and $8 \times 10^{14}$ atoms $/ \mathrm{cm}^{2}$ by choosing a hydrogen temperature in the region between 20 and $50 \mathrm{~K}$. On the other hand, changes in the gas pressure do not affect the target thickness as significantly.

Figure 18 shows a result of such a measurement that was performed at a constant nozzle temperature of $25 \mathrm{~K}$. In the pressure region between 5 and 19 bar, the target thickness increases by less than one order of magnitude. These two measurements indicate the relevance of the variation of the gas parameters e.g. in hadron physics experiments. By adjusting the nozzle temperature one can choose the order of magnitude of the density. Depending on the heating power and the mass of the nozzle holder the variation of the nozzle temperature and the approach to a stable situation is on the order of minutes. Instead, the density of the target can be varied within seconds by changing the pressure of the incoming hydrogen. Therefore, the variation of this parameter is highly suited for a real time adjustment of the target thickness. This might be of interest for adjustments of the luminosity in real time and can be used to compensate for the loss of beam particles in order to achieve a constant luminosity. The fluctuations seen in both figures can be attributed to a change in the emission angle of the cluster beam from the nozzle. Since these fluctuations are reproducible and stable in time it is possible to measure the target thickness as function of the gas pressure and the temperature at the inlet of the nozzle. This mapping can be used for the adjustment of the luminosity. Furthermore, a nozzle tilting system, as it is discussed below, will allow to smooth the observed fluctuations.

Below a nozzle temperature of about $25 \mathrm{~K}$ a brightness structure was observed in the beam, as can be seen in Fig. 7. Surprisingly these structures, which are regions with different cluster 
jet densities, are not symmetrically distributed with respect to the cluster beam axis. Therefore, a dedicated mechanical adjustment system is currently under development [15, 16] which allows to tilt the nozzle axis with respect to the skimmer axis during target operation. Recently, in first tests using a prototype of such a tilting system very high cluster beam densities have been achieved, i.e. approximately $2 \times 10^{15}$ atoms $/ \mathrm{cm}^{3}\left(n_{T} \approx\right.$ $1.6 \times 10^{15}$ atoms $/ \mathrm{cm}^{2}$ ) at a distance of two metres behind the nozzle $\left(p_{0}=18.5 \mathrm{bar}, T_{0}=19 \mathrm{~K}\right)$. This result strengthens the assumption that the decrease of the cluster beam densities at temperature below $25 \mathrm{~K}$ (see Fig. 17) is caused by structures within the cluster jet beam and can be compensated by an online alignment. Detailed systematic investigations on this aspect are currently performed.

\section{Summary}

To perform detailed measurements on cluster beam properties a complete cluster target installation was built in Münster. This provides the possibility to test cluster jet sources in a geometry that matches the one to be used in future experiments such as PANDA/FAIR. By means of a time-of-flight setup based on electron impact ionization, systematic velocity studies on individual clusters are possible. The design and performance of a newly developed cluster jet source was presented. This new cluster source, which is a prototype for the PANDA experiment, is the first one that can provide routinely a hydrogen cluster target thickness of $8 \times 10^{14}$ atoms $/ \mathrm{cm}^{2}$ at a distance of two metres behind the nozzle. Together with the high purity of its target material and the absence of a distinct time structure of the beam, this target is ideally suited for future internal fixed-target experiments at storage rings. Furthermore, an even higher target thickness of $>10^{15}$ atoms $/ \mathrm{cm}^{2}$ has been achieved by a dedicated nozzle tilting system.

\section{Acknowledgements}

The authors would like to thank H. Orth for the very inspiring and helpful discussions and $\mathrm{H}$. Baumeister and W. Hassenmeier for their support during the design of the target device. We are grateful to M. Macri and J. Ritman for providing powerful vacuum pumps. The work provided by the teams of our mechanical and electronic workshops is very much appreciated and we thank them for the excellent manufacturing of the various components. Finally we would like to thank C. Wilkin for suggestions regarding the manuscript. The research project was supported by BMBF (06MS253I and 06MS9149I), GSI F\&E program (MSKHOU1012), EU/FP6 HADRONPHYSICS (506078) and EU/FP7 HADRONPHYSICS2 (227431).

\section{References}

[1] C. Ekström, Nucl. Instr. and Meth. A 362 (1995) 1-15

[2] C. Ekström, et al., Nucl. Instr. and Meth. A 371 (3) (1996) 572-574.

[3] D. Allspach, et al., Nucl. Instr. and Meth. A 410 (2) (1998) 195-205.

[4] H. Dombrowski, et al., Nucl. Instr. and Meth. A 386 (2-3) (1997) 228234.
[5] A. Lehrach, D. Prasuhn, Luminosity Considerations for Internal and External Experiments @ COSY, Annual Report, IKP Forschungszentrum Jülich (2003).

[6] Ö. Nordhage, Ph.D. Thesis, Uppsala Universitet, Sweden, ISSN 16516214, ISBN 91-554-6649-4 (2006).

[7] A. Täschner, et al., AIP Conf. Proc. 950 (2007) 85-88.

[8] E. Knuth, F. Schunemann, J. P. Toennies, J. Chem. Phys. 102 (15) (1995) 6258-6271.

[9] S. General, Diploma Thesis, Westfälische Wilhelms-Universität Münster (2008).

[10] S. Barsov, et al., Nucl. Instr. and Meth. A 462 (2001) 364-381.

[11] H. J. Stein, et al., Phys. Rev. ST-AB 11 (2008) 052801.

[12] PANDA Collaboration, Technical Progress Report - Strong Interaction Studies with Antiprotons, FAIR (February 2005).

[13] A. Täschner, et al., PHN-HSD-PANDA-08, GSI Scientific Report 2010, GSI Report 2011-1, GSI (2010).

[14] A. Khoukaz, et al., Eur. Phys. J. D 5 (2) (1999) 275-281.

[15] A. Khoukaz, Futurejet - Cryogenic Jets of Nano- and Micrometer-Sized Particles for Hadron Physics, HadronPhysics3: Kick-off Meeting CNRS Paris, 17th-18th September 2010, 2010.

[16] A. Khoukaz, Updates on Targets, PANDA Collaboration Meeting, 6th10th June 2011, Protvino, Russia, 2011.

[17] J. Otte, Diploma Thesis, Westfälische Wilhelms-Universität Münster (2007).

[18] A. Täschner, Doctoral Thesis, Westfälische Wilhelms-Universität Münster, Germany (in preparation).

[19] E. Köhler, Diploma Thesis, Westfälische Wilhelms-Universität Münster (2010).

[20] A. Täschner, et al., HK 7.2, DPG Frühjahrstagung, Bonn, Germany, March 15-19, 2010.

[21] J. W. Leachman, et al., J. Phys. Chem. Ref. Data 38 (3) (2009) 721-748.

[22] L. Dick, W. Kubischta, CERN Report (1988), CERN-EP-88-135.

[23] A. Täschner, et al., Annual Report, IKP Forschungszentrum Jülich (2006) 48. 